(A) Check for updates

Cite this: Food Funct., 2017, 8, 2155

\title{
Development of nutraceutical formulations based on the mycelium of Pleurotus ostreatus and Agaricus bisporus
}

\author{
Rossana V. C. Cardoso, ${ }^{a}$ Ângela Fernandes, ${ }^{a, b}$ M. Beatriz P. P. Oliveira, ${ }^{b}$ \\ Ricardo C. Calhelha, ${ }^{a}$ Lillian Barros, (D) *a Anabela Martins ${ }^{a}$ and \\ Isabel C. F. R. Ferreira*a
}

\begin{abstract}
The present work is aimed at developing nutraceutical formulations based on the mycelium of Agaricus bisporus and Pleurotus ostreatus, highlighting the potential of in vitro culture as a tool to improve the production of bioactive compounds, namely phenolic acids and ergosterol. The mycelia of both species were cultured in different solid and liquid media in order to compare the growth rate and yielded biomass. Fruiting bodies, mycelia and culture media were compared regarding the antioxidant activity, anti-inflammatory effects in RAW264.7 cells and cytotoxicity in human tumor cell lines and non-tumor porcine liver cells. P. ostreatus mycelia showed higher contents of ergosterol and phenolic compounds, and stronger antioxidant activity than the corresponding fruiting body. P. ostreatus and A. bisporus did not show anti-inflammatory activity, and $P$. ostreatus was the only one showing cytotoxicity in tumor cell lines. The results show that these mushrooms provide compounds with antioxidant and cytotoxic capacities, with variations among species.
\end{abstract}

Received 3rd April 2017

Accepted 3rd May 2017

DOI: $10.1039 / c 7 f o 00515 f$

rsc.li/food-function logical discoveries over the last few decades, they have become hot research topics.

According to DeFelice ${ }^{2}$ and subsequent references, "a nutraceutical is any substance that is a food or a part of food and provides medical or health benefits, including the prevention and treatment of disease". ${ }^{2}$ Foods, known as "functional foods," are thought to provide benefits beyond basic nutrition and may play a role in reducing or minimizing the risk of certain diseases and other health conditions. ${ }^{3}$ Nutraceuticals and functional characteristics of many traditional foods are being discovered and studied, while new food products are being developed to include beneficial components. By knowing which foods can provide specific health benefits, we can make food and beverage choices that allow us to take greater control of our health.

Agaricus bisporus (J.E. Lange) Imbach and Pleurotus ostreatus (Jacq. Ex Fr.) P. Kumm., belonging to the phylum Basidiomycota, as well as other species of cultivated edible mushrooms, are a good source of natural antioxidants, presenting in their constitution phenolic compounds, especially phenolic acids, followed by tocopherols (mainly $\alpha$-tocopherol), ascorbic acid and carotenoids, especially $\beta$-carotene. ${ }^{4,5}$

A. bisporus, known as "champignon", "white mushroom" or "white button mushroom" has demonstrated valuable medicinal properties including antitumor, anti-aromatase, antimicrobial, immunomodulatory, anti-inflammatory and antioxidant activities. ${ }^{6}$ P. ostreatus, better known as "oyster mush-

\footnotetext{
${ }^{a}$ Centro de Investigação de Montanha (CIMO), ESA, Instituto Politécnico de Bragança, Campus de Santa Apolónia, 5300-253 Bragança, Portugal. E-mail: lillian@ipb.pt, iferreira@ipb.pt; Fax:+351273325405; Tel: +351273303285, 351273303219

${ }^{b}$ REQUIMTE/LAQV, Faculdade de Farmácia, Universidade do Porto, Rua Jorge Viterbo Ferreira no. 228, 4050-313 Porto, Portugal
} 
room" or "repolgas" is among the most consumed mushrooms all over the world, due to its easy production and quick growth but also due to its organoleptic, nutritional and medicinal properties. $^{7}$ The latter include immunologic, antitumor, antiinflammatory and antimicrobial activities, among others. ${ }^{8,9}$

The great majority of the studies reporting mushroom properties are conducted with the fruiting bodies, but culture media used in mushroom cultivation have also been explored as potential sources of bioactive compounds. ${ }^{10}$ On the other hand, the in vitro culture of mycelia is becoming a promising alternative to obtain sources of bioactive compounds, mainly due to the shorter incubation time and easier culture conditions (less space needed, low probability of contamination and higher biomass yields when compared with fruiting bodies). ${ }^{11}$

The fruiting bodies and mycelia of A. bisporus and $P$. ostreatus were previously studied for their chemical composition, and antioxidant, anti-inflammatory and cytotoxic activities, after being harvested at different periods. ${ }^{42-17}$ However, the present study goes further and compares fruiting bodies, mycelia and culture media in terms of bioactive properties and compounds. Thereby, the main objective was to develop nutraceutical formulations based on the fruiting body and mycelium of $A$. bisporus and P. ostreatus, as also the corresponding culture media, highlighting the potential of in vitro culture as a tool to improve the production of bioactive compounds by two different mushroom species.

\section{Materials and methods}

\subsection{Samples and mycelium production}

Agaricus bisporus (J.E. Lange) Imbach was bought from a local food store and Pleurotus ostreatus (Jacq. ex Fr.) P. Kumm. was obtained from a sterile wheat grain and boiled wheat straw cultivation bale in Bragança.

Mycelia from each of the mushrooms were isolated from sporocarps in two solid and liquid media: (1) modified MelinNorkrans solid (incomplete medium with agar and without micronutrients, casamino acids and malt extract) (MMNi solid) and liquid medium (incomplete medium without agar, micronutrients, casamino acids and malt extract) (MMNi liquid); (2) Potato Dextrose Agar (PDA) solid medium and Potato Dextrose Broth (PDB) liquid medium. ${ }^{18,19}$

The strains were maintained on Petri dishes $(9 \mathrm{~cm}$ diameter) containing $10 \mathrm{~mL}$ of solid media and flasks with $20 \mathrm{~mL}$ of medium at $25{ }^{\circ} \mathrm{C}$ in the dark and cultured until mycelium covered most of the medium: approximately 21 days for $P$. ostreatus in solid medium and 42 days in liquid medium; and 56 days for $A$. bisporus in solid and liquid media. Radial growth measurements were registered every week from the inoculation time until the full growth of the mycelium (covering all the available area). ${ }^{18,19}$ After different growth times mycelia were recovered from both solid and liquid media. Fruiting bodies and mycelia were lyophilized (FreeZone 4.5, Labconco, MO, USA) and ground to a fine powder (20 mesh).

\subsection{Standards and reagents}

The solvents acetonitrile $99.9 \%$ and methanol were of highperformance liquid chromatography (HPLC) grade from LabScan (Lisbon, Portugal). The standards of ergosterol, phenolic compounds (gallic, protocatechuic, $p$-hydroxybenzoic and $p$-coumaric acids), cinnamic acid and trolox (6-hydroxy-2,5,7,8tetramethylchroman-2-carboxylic acid) were purchased from Sigma (St Louis, MO, USA). 2,2-Diphenyl-1-picrylhydrazyl (DPPH) was obtained from Alfa Aesar (Ward Hill, MA, USA). Dulbecco's modified Eagle's medium, Hank's balanced salt solution (HBSS), fetal bovine serum (FBS), L-glutamine, trypsinEDTA, and penicillin/streptomycin solution $\left(100 \mathrm{U} \mathrm{mL}^{-1}\right.$ and $100 \mathrm{mg} \mathrm{mL} \mathrm{m}^{-1}$, respectively) were purchased from Gibco Invitrogen Life Technologies (Paisley, UK). Sulforhodamine B, trypan blue, trichloroacetic acid (TCA), lipopolysaccharide (LPS) and Tris were purchased from Sigma Chemical Co. (Saint Louis, MO, USA). RAW264.7 cells were purchased from ECACC (European Collection of Animal Cell Culture, Salisburg, UK), and DMEM medium from HyClone (GE Healthcare Life Sciences, Logan, UT, USA). The Griess Reagent System Kit was purchased from Promega, and dexamethasone from Sigma. Thiamine, casamino acids, malt extract and agar were obtained from Panreac Applichem (Barcelona, Spain). PDA and PDB were acquired from Oxoid microbiology products (Hampshire, United Kingdom). All other reagents and solvents were of analytical grade and obtained from common sources. Water was treated in a Milli-Q water purification system (TGI Pure Water Systems, Greenville, SC, USA).

\subsection{Preparation of the extracts}

Dried powder of each sample was placed in a beaker $(\approx 2 \mathrm{~g})$ and was extracted by magnetic stirring with methanol $(30 \mathrm{~mL})$ at $25{ }^{\circ} \mathrm{C}$ and $150 \mathrm{rpm}$, for $1 \mathrm{~h}$. The extract was separated from the residue by filtration through Whatman paper No. 4 into a round flask. The residue was re-extracted once more under the same conditions and the filtrates were combined and concentrated with a rotary evaporated at $\approx 40{ }^{\circ} \mathrm{C}$ (rotary evaporator, Büchi, Flawil Switzerland). The extraction yield was calculated by measuring the extract weight in relation to the initial mass sample and stock solutions were prepared for the subsequent bioactive assays. ${ }^{12}$ For phenolic and ergosterol compositions the extracts were dissolved in methanol at a concentration of $20 \mathrm{mg} \mathrm{mL}{ }^{-1}$ and filtered through a $0.22 \mu \mathrm{m}$ nylon disposable filter.

\subsection{Chemical characterization}

2.4.1. In terms of phenolic acids. The phenolic acid analysis was performed using a Shimadzu 20A series ultra-fast liquid chromatograph (UFLC, Shimadzu Corporation, Kyoto, Japan) coupled with a diode array detector. ${ }^{12}$ The phenolic acids were identified by comparison with phenolic acid standards and quantified by comparing the area of their peaks recorded at $280 \mathrm{~nm}$ with calibration curves $\left(5-100 \mu \mathrm{g} \mathrm{mL} \mathrm{mL}^{-1}\right)$ obtained from commercial standards. The results were expressed as $\mu \mathrm{g}$ per $\mathrm{g}$ of extract. 
2.4.2. In terms of ergosterol. Ergosterol analysis was performed by high performance liquid chromatography coupled with an ultraviolet detector (HPLC-UV). The detection was performed at $285 \mathrm{~nm}$ and data were analyzed using Clarity 2.4 Software (DataApex). Ergosterol was quantified by comparing the area of its peak with the calibration curve obtained from a commercial standard. The results were expressed in $\mathrm{mg}$ per $\mathrm{g}$ of extract. ${ }^{20}$

\subsection{Evaluation of the bioactive properties}

2.5.1. Antioxidant activity. The crude extracts were dissolved in methanol obtaining stock solutions $\left(20-80 \mathrm{mg} \mathrm{mL}^{-1}\right)$, and then subjected to serial dilutions (40 and $0.02 \mathrm{mg} \mathrm{mL}^{-1}$ ), depending on the stock solutions and according to the assay. The in vitro antioxidant activity of the extracts was evaluated by performing four different assays: DPPH radical-scavenging activity, reducing power, $\beta$-carotene bleaching inhibition and thiobarbituric acid reactive substance (TBARS) assay. ${ }^{21,22}$ Trolox was used as the positive control and the results were expressed as $\mathrm{EC}_{50}$ values (sample concentration that achieved $50 \%$ of antioxidant activity or 0.5 absorbance in reducing power assay).

2.5.2. Anti-inflammatory activity. For the anti-inflammatory activity assay, the methanolic extracts were dissolved in water at a concentration of $10 \mathrm{mg} \mathrm{mL}^{-1}$. For the various assays, the extracts were then subjected to further dilutions from $10 \mathrm{mg} \mathrm{mL} \mathrm{m}^{-1}$ to $0.16 \mathrm{mg} \mathrm{mL}^{-1}$. Dexametazona was used as the positive control. The mouse macrophage-like cell line RAW 264.7 was used with a view to assessing the anti-inflammatory activity following a procedure previously reported. ${ }^{15}$ For the determination of nitric oxide, a Griess Reagent System kit was used. The nitric oxide produced was determined by measuring the absorbance at $540 \mathrm{~nm}$ (microplate reader ELX800 Biotek). The percentage of inhibition of the NO production was calculated for each sample concentration. The results were expressed as percent inhibition of NO production compared to the negative control, and $\mathrm{EC}_{50}$ values (concentration of the sample that provides $50 \%$ inhibition of NO production) were also estimated. ${ }^{15}$

2.5.3. Cytotoxic activity. For the cytotoxic activity assay, the methanolic extracts were dissolved in water at a concentration of $10 \mathrm{mg} \mathrm{mL} \mathrm{m}^{-1}$. For the various assays, the extracts were then subjected to further dilutions from $10 \mathrm{mg} \mathrm{mL}^{-1}$ to 0.16 $\mathrm{mg} \mathrm{mL}^{-1}$. Ellipticine was used as the positive control. Four human tumor cell lines were used: MCF-7 (breast adenocarcinoma), NCI-H460 (non-small cell lung cancer), HeLa (cervical carcinoma) and HepG2 (hepatocellular carcinoma). The cell density determination was performed using a sulforhodamine B assay. The absorbance was measured at $540 \mathrm{~nm}$ and the results were expressed as $\mathrm{GI}_{50}$ values (sample concentration that inhibited $50 \%$ of the net cell growth) in $\mu \mathrm{g}$ per $\mathrm{mL} .^{23}$ For evaluation of the cytotoxicity in non-tumor cells, a cell culture from freshly harvested porcine liver and obtained from a local slaughterhouse was prepared according to the procedure described by Abreu et al. ${ }^{24}$ and referred to as PLP2. The sulforhodamine B colorimetric assay was also followed.

\subsection{Statistical analysis}

For each culture component, fruiting body, mycelium and culture medium, three independent samples were analysed and all assays were carried out in triplicate. Data were expressed as mean \pm standard deviation. All statistical tests were performed at a 5\% significance level using IBM SPSS Statistics for Windows, version 23.0 (IBM Corp., Armonk, NY, USA). The fulfilment of the one-way ANOVA requirements, specifically the normal distribution of the residuals (data not shown) and the homogeneity of variance, was tested by means of Shapiro Wilk's and Levene's tests, respectively. All dependent variables were compared using Tukey's honestly significant difference (HSD) or Tamhane's T2 multiple comparison tests, when homoscedasticity was verified or not, respectively.

\section{Results and discussion}

\subsection{Mycelia production}

The establishment of $P$. ostreatus and A. bisporus in vitro allowed the evaluation of differential growth rates and chemical characterization in two culture media and under two different physical conditions (solid vs. liquid). In vitro production of mycelium can help to overcome difficulties associated with mushroom's availability related to its seasonality or adverse climatic conditions, being an important methodology for continuous production of mycelia with uniform characteristics, contaminant free and of high quality for food and pharmaceutical purposes. ${ }^{25}$

At the industrial level, the mycelia growth rate and produced biomass are of extreme importance and both indicators are shown in Fig. 1 and 2, respectively. As it can be observed, after 7 days, the species $P$. ostreatus showed good growth on both media (PDA and MMNi solid medium). Mycelia were depigmented and cottony, and in PDA mycelia appeared as a thin layer. The mycelia showed faster radial growth on PDA and a higher biomass than on solid MMNi (fresh and dry weight). At the end of 3 weeks the entire plate was already covered with mycelia and the growth remained constant.

However, in liquid PDB and MMNi mycelia started growing at day 8 , and presented the same characteristics than in solid media. In four weeks some of the mycelia already covered the PDB medium plate, but in liquid MMNi they stopped growing and did not cover the plate, presenting half of the diameter of PDB. In PDB liquid medium the P. ostreatus mycelia had a higher growth rate and biomass than in liquid $\mathrm{MMNi}$ (fresh and dry weight). A. bisporus mycelia showed little growth in all media (PDA, PDB, solid and liquid MMNi). This species presented depigmented and rhizomorphic mycelia. This species started growing in solid medium after 7 days. The mycelia grew in very small diameters and also grew into the culture medium, which made it difficult to collect the mycelia; to solve this problem, mycelia were cultivated with cellophane paper.

The radial growth was faster in MMNi than in PDA, despite the similarities in the produced biomass (fresh and dry weight). They stopped growing within 6 weeks and did not 
A

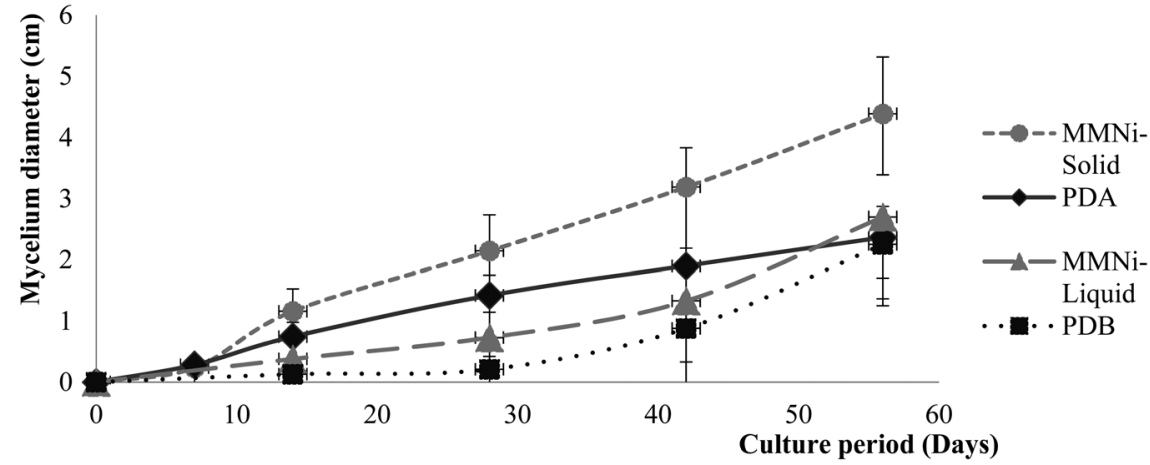

B

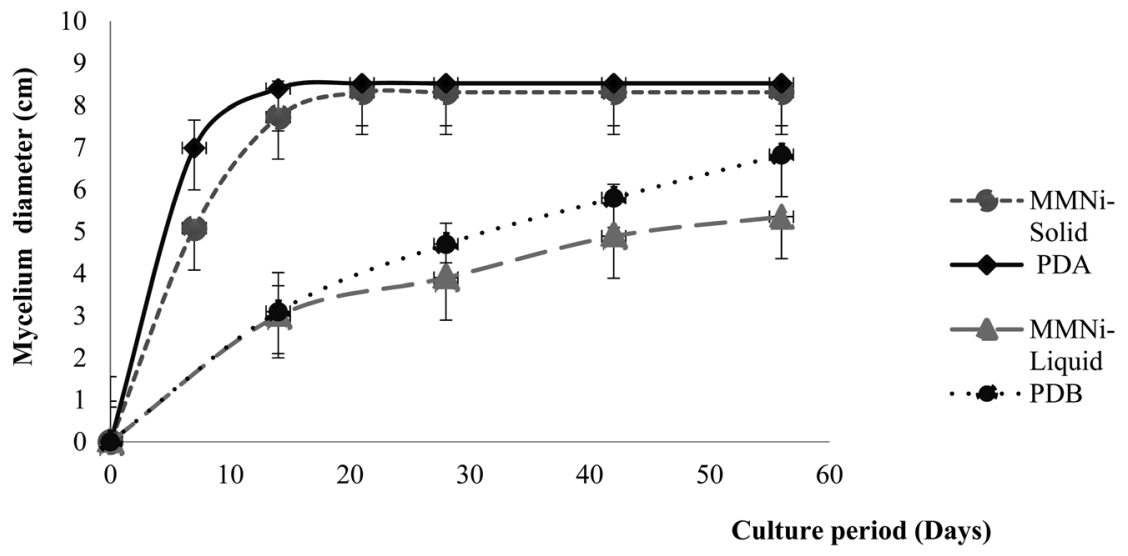

Fig. 1 Radial growth of $A$. bisporus (A) and P. ostreatus (B) mycelia cultivated in different culture media throughout time.

cover the plate. In MMNi liquid medium they started growing from day 14, but in PDB they started just 1 week after MMNi. To stimulate growth, agitation was performed and, as a result the mycelium grew as small grains. The radial growth and fresh weight biomass were higher in MMNi than in PDB, although in PDB it showed higher dry weight biomass. They stopped growing within 8 weeks and didn't cover the plate.

The main distinctive feature considering both radial growth patterns is that $P$. ostreatus exhibited very fast initial growth in both media, although slower under liquid conditions reaching maximum radial growth after 14 days in solid medium while A. bisporus showed a slower and continuous growth rate along the 56 day period of culture, never reaching the complete top covering of the media. Although radial growth was higher in solid than in liquid media for both species, biomass production was higher in liquid than in solid media being similar in composition of both media for A. bisporus but better in PDB for $P$. ostreatus and not very far from the biomass production in solid PDA medium.

Under the culture conditions studied here, we can consider better growth results in PDA and PDB media for P. ostreatus with similar biomass production. A. bisporus exhibited similar biomass production in both media formulations but significantly higher under liquid conditions. After these growth results, chemical characterization of the samples will determine the best growth conditions for our main purposes.

\subsection{Chemical characterization of the extracts}

Mycelia, culture media (liquid culture media in which mycelia were grown) and fruiting bodies were compared in terms of bioactive compounds and properties.

The phenolic acid and ergosterol contents are presented in Table 1. The highest content of ergosterol was found in the fruiting body of $A$. bisporus (17.4 $\pm 0.1 \mathrm{mg} \mathrm{g}^{-1}$ extract); as expected, it was not found in the culture media.

However, for $P$. ostreatus it was the mycelium cultivated in the PDB medium that showed a higher quantity (18.56 \pm $0.07 \mathrm{mg} \mathrm{g}^{-1}$ extract). The analysis of ergosterol in these mushroom species was previously conducted by Villares et al. ${ }^{26}$ No ergosterol was also found in the PDB culture medium.

In general and comparing mycelia, $A$. bisporus showed lower contents of ergosterol (ranging from MMNi solid $-2.87 \pm 0.04$ to $\mathrm{PDB}-3.71 \pm 0.04 \mathrm{mg} \mathrm{g}^{-1}$ extract) than $P$. ostreatus (ranging from MMNi liquid $-9.08 \pm 0.03$ to PDB $-18.56 \pm 0.07 \mathrm{mg} \mathrm{g}^{-1}$ extract). But in fruiting bodies, it was A. bisporus $\left(17.4 \pm 0.1 \mathrm{mg} \mathrm{g}^{-1}\right.$ extract) that showed a higher content of ergosterol in comparison with $P$. ostreatus $\left(9.7 \pm 0.2 \mathrm{mg} \mathrm{g}^{-1}\right.$ extract).

Among the phenolic acids, $p$-hydroxybenzoic and cinnamic acids were the major compounds in both mushrooms and in all samples. $p$-Hydroxybenzoic acid in mycelium grown in PDA reached quantities nearly five times higher in $A$. bisporus $\left(1030 \pm 59 \mu \mathrm{g} \mathrm{g}{ }^{-1}\right.$ extract $)$ than in P. ostreatus $\left(188 \pm 34 \mu \mathrm{g} \mathrm{g}^{-1}\right.$ 


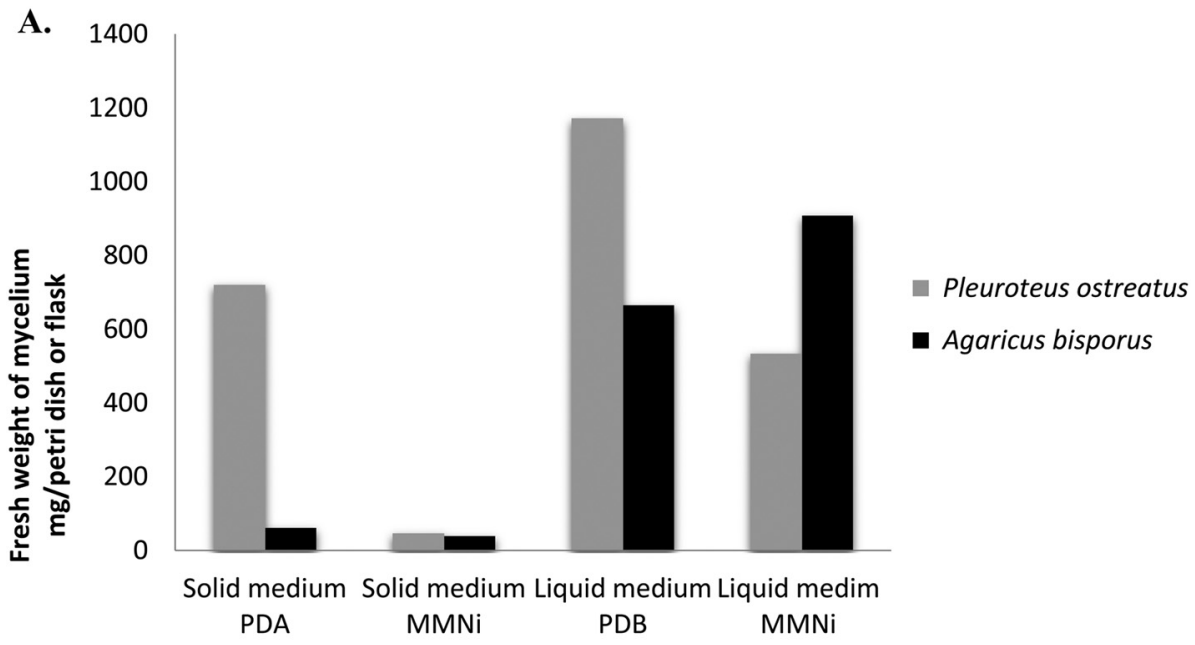

B.

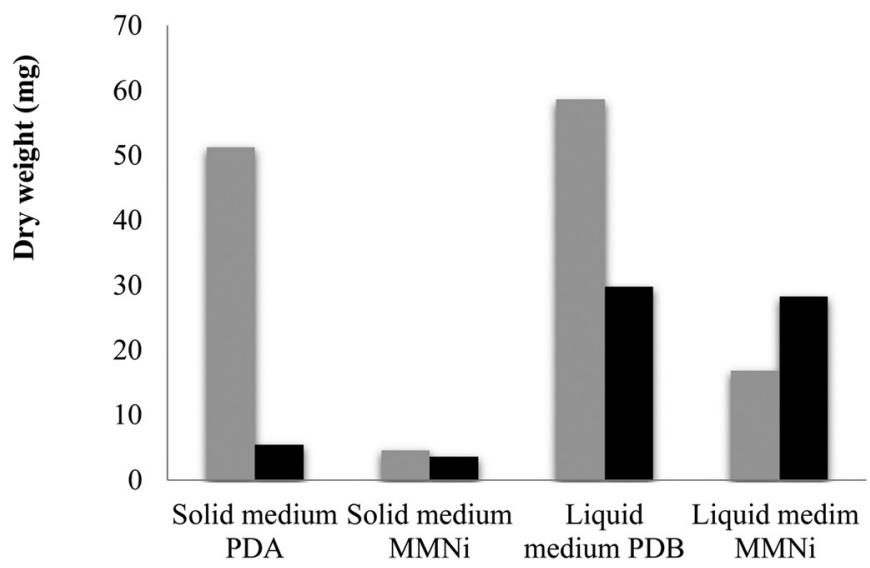

Pleuroteus ostreatus

- Agaricus bisporus

Fig. 2 Total biomass of $A$. bisporus (A) and P. ostreatus (B) mycelia.

Table 1 Ergosterol ( $\mathrm{mg} \mathrm{g}^{-1}$ extract) and phenolic acid ( $\mu \mathrm{g} \mathrm{g}^{-1}$ extract) contents in the mycelia and culture media of $A$. bisporus and $P$. ostreatus

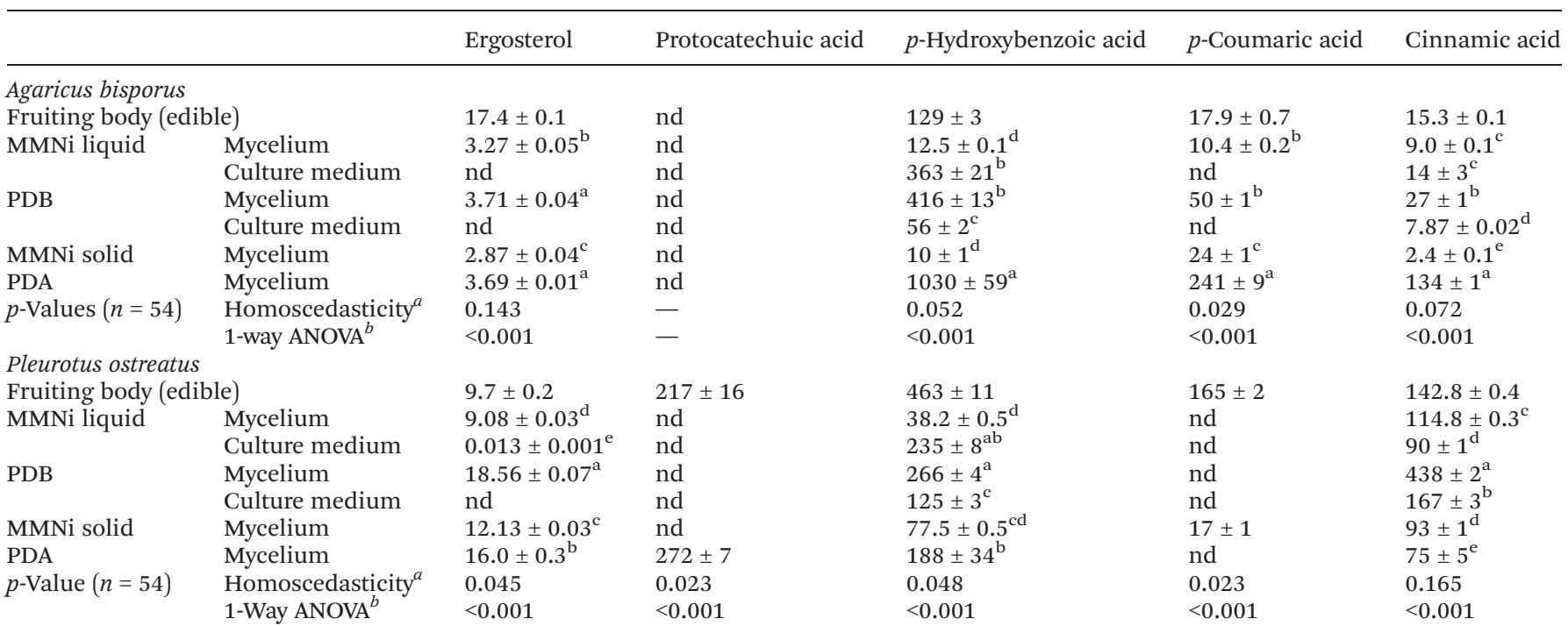

nd - Not detected. The values corresponding to the fruiting body of both mushrooms (edible samples) are presented. Values are given as mean \pm standard deviation. ${ }^{a}$ Homoscedasticity among culture components was tested by the Levene test: homoscedasticity, $p>0.05$; heteroscedasticity, $p<0.05 .{ }^{b} p<0.05$ indicates that the mean value of at least one component differs from the others (in this case, multiple comparison tests were performed). For each culture component, means within a column with different letters differ significantly $(p<0.05)$. 
extract). The $P$. ostreatus fruiting body also showed high levels of this phenolic acid.

The phenolic compounds obtained in the present study are the same as those reported in the literature for A. bisporus and $P$. ostreatus (except the vanillic acid that could not be found in the present study). ${ }^{4}$ Cinnamic acid was found in the highest amount in $P$. ostreatus mycelium grown on $\mathrm{PDB}\left(438 \pm 2 \mu \mathrm{g} \mathrm{g}^{-1}\right.$ extract).

Regarding fruiting bodies, A. bisporus and $P$. ostreatus showed higher levels of $p$-hydroxybenzoic acid (129 \pm 3 and $463 \pm 11 \mu \mathrm{g} \mathrm{g}^{-1}$ extract, respectively) than those of the other phenolic acids identified.

A. bisporus showed a higher quantity of cinnamic acid in the mycelium produced in PDA culture medium $\left(134 \pm 1 \mathrm{mg} \mathrm{g}^{-1}\right.$ extract), while $P$. ostreatus showed the highest concentration in the mycelium produced in PDB ( $438 \pm 2 \mathrm{mg} \mathrm{g}^{-1}$ extract).

Protocatechuic acid was not found in A. bisporus like in previous studies ${ }^{12}$ and in $P$. ostreatus it was only found in the fruiting body $\left(217 \pm 16 \mathrm{mg} \mathrm{g}^{-1}\right.$ extract $)$ and in the mycelium produced in PDA $\left(272 \pm 7 \mathrm{mg} \mathrm{g}^{-1}\right.$ extract). In previous studies protocatechuic acid in $P$. ostreatus was found only in the fruiting body. ${ }^{12}$

A. bisporus showed higher levels of $p$-coumaric acid (mycelium PDA - $241 \pm 9 \mathrm{mg} \mathrm{g}^{-1}$ extract) when compared with P. ostreatus. This phenolic acid was present in all samples of A. bisporus, except in the culture media. But for $P$. ostreatus, it only appeared in the fruiting body (165 $\pm 2 \mathrm{mg} \mathrm{g}^{-1}$ extract) and in the mycelium produced in MMNi solid medium (17 $\pm 1 \mathrm{mg} \mathrm{g}^{-1}$ extract).

The differences observed between the studied samples and the values reported in the literature are related to their genetic variability, since they are different strains, from different harvesting years and geographic locations that also comprise diverse climatic conditions, which all have an influence on the chemical composition. ${ }^{4,12}$ For example, Reis et al. ${ }^{12}$ described the presence of another phenolic acid (gallic acid) in the same mushroom species from cultivated origin. The phenolic compounds and ergosterol contents cannot be directly compared to previous studies because our results are expressed in $\mathrm{mg} \mathrm{g}^{-1}$ extract, and other literature reports expressed the results on the dry weight basis $\left(\mathrm{mg} \mathrm{g}^{-1} \mathrm{dw}\right) .{ }^{4,12,26}$

\subsection{Antioxidant activity}

The organisms are equipped against damage caused by free radicals with several defense mechanisms acting in different ways. As indicated, the antioxidants are natural molecules which prevent the uncontrolled formation of free radicals and ROS or inhibit their reaction with biological structures, interrupting the chain reaction and forming radicals having low reactivity which are easily removed from the body. ${ }^{22,27}$

Maintaining the balance between the production of free radicals and antioxidant cell defenses is an essential condition for the normal functioning of the body. ${ }^{5}$ However, a balance between ROS and antioxidant defenses can be destabilized either by overproduction of ROS or the loss of the antioxidant defenses of cells. This imbalance is known as oxidative stress and, in this case, the excess ROS oxidize and can damage lipids, proteins and DNA of the cell, leading to alteration and inhibiting its normal function. ${ }^{5}$

The results obtained for each of the performed antioxidant activity assays, expressed as $\mathrm{EC}_{50}$ values, are shown in Table 2.

Regarding fruiting bodies, the highest activity was obtained in the TBARS formation inhibition assay (A. bisporus: $0.09 \pm$ $0.01 \mathrm{mg} \mathrm{mL} \mathrm{m}^{-1}$; . ostreatus: $\left.0.090 \pm 0.002 \mathrm{mg} \mathrm{mL}^{-1}\right)$, followed

Table 2 Antioxidant activity $\left(\mathrm{EC}_{50}\right.$ values, $\left.\mathrm{mg} \mathrm{mL}^{-1}\right)$ of the mycelia and culture media of $A$. bisporus and $P$. ostreatus

\begin{tabular}{|c|c|c|c|c|c|}
\hline & & $\begin{array}{l}\text { DPPH scavenging } \\
\text { activity }\end{array}$ & $\begin{array}{l}\text { Reducing } \\
\text { power }\end{array}$ & $\begin{array}{l}\beta \text {-Carotene bleaching } \\
\text { inhibition }\end{array}$ & $\begin{array}{l}\text { TBARS formation } \\
\text { inhibition }\end{array}$ \\
\hline \multicolumn{6}{|l|}{ Agaricus bisporus } \\
\hline Fruiting body (edible) & & $3.27 \pm 0.03$ & $0.81 \pm 0.01$ & $2.2 \pm 0.1$ & $0.09 \pm 0.01$ \\
\hline \multirow[t]{2}{*}{ MMNi liquid } & Mycelium & $17.7 \pm 0.3^{\mathrm{b}}$ & $1.98 \pm 0.01^{\mathrm{c}}$ & $4.4 \pm 0.2^{\mathrm{ab}}$ & $0.7 \pm 0.1^{\mathrm{b}}$ \\
\hline & Culture medium & $18.2 \pm 0.5^{\mathrm{b}}$ & $2.25 \pm 0.02^{\mathrm{a}}$ & $4.7 \pm 0.1^{\mathrm{ab}}$ & $1.4 \pm 0.1^{\mathrm{a}}$ \\
\hline \multirow[t]{2}{*}{ PDB } & Mycelium & $14.7 \pm 0.1^{\mathrm{c}}$ & $1.23 \pm 0.01^{\mathrm{e}}$ & $4.2 \pm 0.4^{\mathrm{b}}$ & $0.19 \pm 0.01^{\mathrm{cd}}$ \\
\hline & Culture medium & $21.8 \pm 0.6^{\mathrm{a}}$ & $2.11 \pm 0.02^{\mathrm{b}}$ & $4.9 \pm 0.1^{\mathrm{a}}$ & $0.36 \pm 0.02^{\mathrm{c}}$ \\
\hline MMNi solid & Mycelium & $13.31 \pm 0.1^{\mathrm{d}}$ & $1.58 \pm 0.01^{d}$ & $4.5 \pm 0.1^{\mathrm{ab}}$ & $0.22 \pm 0.003^{\mathrm{cd}}$ \\
\hline PDA & Mycelium & $10.1 \pm 0.03^{\mathrm{e}}$ & $0.46 \pm 0.01^{\mathrm{f}}$ & $4.2 \pm 0.1^{\mathrm{b}}$ & $0.13 \pm 0.003^{\mathrm{d}}$ \\
\hline \multirow[t]{2}{*}{$p$-Value $(n=54)$} & Homoscedasticity $^{a}$ & 0.005 & 0.174 & 0.045 & 0.002 \\
\hline & 1-Way ANOVA $^{b}$ & $<0.001$ & $<0.001$ & $<0.001$ & $<0.001$ \\
\hline \multicolumn{6}{|l|}{ Pleurotus ostreatus } \\
\hline Fruiting body (edible) & & $3.2 \pm 0.1$ & $1.79 \pm 0.01$ & $0.7 \pm 0.1$ & $0.09 \pm 0.002$ \\
\hline \multirow[t]{2}{*}{ MMNi liquid } & Mycelium & $56.9 \pm 0.6^{\mathrm{a}}$ & $4.08 \pm 0.06^{\mathrm{a}}$ & $4.6 \pm 0.2^{\mathrm{a}}$ & $0.220 \pm 0.001^{\mathrm{a}}$ \\
\hline & Culture medium & $57.3 \pm 0.9^{\mathrm{a}}$ & $3.66 \pm 0.01^{\mathrm{b}}$ & $2.1 \pm 0.1^{\mathrm{b}}$ & $0.22 \pm 0.01^{\mathrm{a}}$ \\
\hline \multirow[t]{2}{*}{ PDB } & Mycelium & $16.87 \pm 0.09^{d}$ & $1.85 \pm 0.01^{\mathrm{c}}$ & $0.70 \pm 0.06^{\mathrm{d}}$ & $0.04 \pm 0.0004^{\mathrm{d}}$ \\
\hline & Culture medium & $32.3 \pm 0.6^{\mathrm{c}}$ & $1.9 \pm 0.1^{\mathrm{c}}$ & $1.5 \pm 0.1^{\mathrm{c}}$ & $0.17 \pm 0.01^{\mathrm{b}}$ \\
\hline MMNi solid & Mycelium & $52.4 \pm 1.4^{\mathrm{b}}$ & $3.96 \pm 0.04^{\mathrm{a}}$ & $2.4 \pm 0.1^{\mathrm{b}}$ & $0.18 \pm 0.01^{\mathrm{b}}$ \\
\hline PDA & Mycelium & $17.1 \pm 0.4^{\mathrm{d}}$ & $1.94 \pm 0.01^{\mathrm{c}}$ & $0.92 \pm 0.01^{\mathrm{d}}$ & $0.080 \pm 0.001^{\mathrm{c}}$ \\
\hline \multirow[t]{2}{*}{$p$-Value $(n=54)$} & Homoscedasticity $^{a}$ & 0.059 & 0.108 & 0.016 & 0.003 \\
\hline & 1-Way $\mathrm{ANOVA}^{b}$ & $<0.001$ & $<0.001$ & $<0.001$ & $<0.001$ \\
\hline Trolox & & $41.68 \pm 0.28$ & $41.43 \pm 1.27$ & $18.21 \pm 1.12$ & $22.84 \pm 0.74$ \\
\hline
\end{tabular}

nd - Not detected. The values corresponding to the fruiting body of both mushrooms (edible samples) are presented. Values are given as mean \pm standard deviation. ${ }^{a}$ Homoscedasticity among culture components was tested by the Levene test: homoscedasticity, $p>0.05$; heteroscedasticity, $p<0.05 .{ }^{b} p<0.05$ indicates that the mean value of at least one component differs from the others (in this case, multiple comparison tests were performed). For each culture component, means within a column with different letters differ significantly $(p<0.05)$. 
by $\beta$-carotene bleaching inhibition (in the case of $P$. ostreatus: $0.7 \pm 0.1 \mathrm{mg} \mathrm{mL} \mathrm{mL}^{-1}$ ) or reducing power (in the case of A. bisporus: $0.81 \pm 0.01 \mathrm{mg} \mathrm{mL}^{-1}$ ). The lowest activity was observed in DPPH radical scavenging activity assay ( $P$. ostreatus: $3.2 \pm 0.1 \mathrm{mg} \mathrm{mL}^{-1}$; A. bisporus: $\left.3.27 \pm 0.03 \mathrm{mg} \mathrm{mL}^{-1}\right)$.

In general, the values obtained for $A$. bisporus (for example: reducing power $-0.81 \pm 0.01 \mathrm{mg} \mathrm{mL}^{-1}$ and TBARS $-0.09 \pm$ $0.01 \mathrm{mg} \mathrm{mL}^{-1}$ ) and P. ostreatus (for example: $\beta$-carotene $-0.7 \pm$ $0.1 \mathrm{mg} \mathrm{mL}^{-1}$ and TBARS- $0.09 \pm 0.002 \mathrm{mg} \mathrm{mL} \mathrm{m}^{-1}$ ) fruiting bodies represent higher antioxidant activity than that reported previously (for example: A. bisporus: reducing power $-1.80 \pm$ $0.03 \mathrm{mg} \mathrm{mL}{ }^{-1}$ and TBARS $-2.9 \pm 0.5 \mathrm{mg} \mathrm{mL}^{-1}$; P. ostreatus: $\beta$-carotene $-2.7 \pm 0.2 \mathrm{mg} \mathrm{mL}^{-1}$ and TBARS $-2.6 \pm 0.9$ $\mathrm{mg} \mathrm{mL}^{-1}$ ) (except for DPPH in A. bisporus). ${ }^{12}$ Also, for mycelia, the antioxidant activity of these species was higher (for example: A. bisporus: DPPH $-10.1 \pm 0.03 \mathrm{mg} \mathrm{mL}^{-1}$; P. ostreatus: $\mathrm{DPPH}-17.1 \pm 0.4 \mathrm{mg} \mathrm{mL}{ }^{-1}$ ) (except for $\beta$-carotene bleaching inhibition assay in $A$. bisporus) than that reported by those

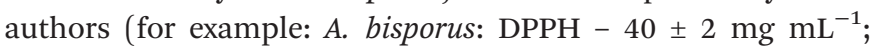
P. ostreatus: $\left.\mathrm{DPPH}-29 \pm 6 \mathrm{mg} \mathrm{mL}{ }^{-1}\right) .{ }^{12}$

In this context, phenolic acids are described as important compounds with antioxidant activity in mushrooms due to the presence of $\mathrm{OH}$ groups in their chemical structures that are known for their ability to scavenge free radicals. ${ }^{28}$

Regarding $P$. ostreatus, the DPPH scavenging activity and $\beta$-carotene bleaching inhibition $(3.2 \pm 0.1$ and $0.7 \pm 0.1$ $\mathrm{mg} \mathrm{mL}^{-1}$, respectively) were higher than those previously reported $\left(5.11 \pm 0.65\right.$ and $\left.440 \pm 43 \mathrm{mg} \mathrm{mL}^{-1}\right)$, respectively. ${ }^{17}$ A. bisporus also showed higher radical scavenging activity $\left(3.27 \pm 0.03 \mathrm{mg} \mathrm{mL}^{-1}\right)$ and reducing power $\left(0.81 \pm 0.01 \mathrm{mg} \mathrm{mL}^{-1}\right)$ than a similar sample previously reported in the literature (9.61 $\pm 0.07 \mathrm{mg} \mathrm{mL}^{-1}$ and $3.63 \pm 0.02 \mathrm{mg} \mathrm{mL}^{-1}$, respectively). ${ }^{14}$

Considering the main purpose of this work, it was very interesting to discover that the antioxidant activity measured in the mycelia and in culture media was very close (in some cases better) to that verified in the fruiting bodies, emphasizing the high potential of these in vitro culture produced fungal components. In the case of the culture media, these results have shown increased interest, since those components are usually considered as by-products of mushroom's cultivation. Furthermore, the differences among the same culture media, after being used to grow each one of the mushroom species, indicate that the evaluated antioxidant activity is in fact due to the mycelium, grown in it, and not due to the culture media components. Therefore, some compounds responsible for the antioxidant activity are released into the culture medium, while others are maintained in the mycelium.

The culture media of $P$. ostreatus gave better results than the same components of $A$. bisporus. P. ostreatus in PDB culture medium showed higher $\beta$-carotene bleaching inhibition, TBARS formation inhibition and reducing power, while A. bisporus in MMNi culture medium gave better results in DPPH scavenging activity and reducing power assays. On the other hand, A. bisporus mycelia produced in liquid medium MMNi gave the worst results compared to $P$. ostreatus in TBARS formation inhibition and $\beta$-carotene bleaching inhibition assays.

\subsection{Anti-inflammatory activity}

The ability of both mushroom species to modulate the production of the inflammatory mediator NO (nitric oxide) was evaluated in RAW 264.7 macrophages by pre-treating cells with various concentrations of their methanolic extracts (Table 3).

LPS (lipopolysaccharide) is a cell wall component of Gramnegative bacteria, and plays a central role in the pathogenesis of several diseases. ${ }^{15}$ Macrophages are the main components of the innate immune system, and have essential regulation functions under several immunopathological conditions during the inflammatory process. ${ }^{29}$

When macrophages are exposed to LPS, the LPS binds to receptors that activate several signaling pathways which in

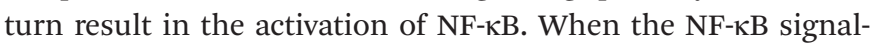
ing pathway is activated, macrophages secrete NO and proinflammatory cytokines. Hence, natural agents that regulate the production of various cytokines and suppress the overproduction of NO may have protective roles in inflammationrelated diseases such as cancer, diabetes and cardiovascular disease. ${ }^{30,31}$ Endotoxin LPS is able to induce the production of mediators like NO, pro-inflammatory cytokines (besides inhibiting anti-inflammatory cytokines) and tumor necrosis factor in macrophages. Therefore, macrophages stimulated by LPS have been widely used for evaluation of anti-inflammatory activities in vitro. ${ }^{32}$ In addition, due to the reproducible response of RAW264.7 macrophages to LPS, this cell line has been widely used for inflammatory research. ${ }^{15}$

Although phenolic acids were the compounds identified in the studied species, their content did not correlate with this bioactivity, which suggests that the contribution of this kind of compound to the anti-inflammatory activity is not relevant.

However, cinnamic acid derivatives, such as caffeic acid, have been previously described to down-regulate the production of proinflammatory cytokines in CACO-2 cells. ${ }^{13}$ In contrast, in other studies, cinnamic acid derivatives, such as caffeic acid, chlorogenic acid, ferulic acid and $p$-hydroxybenzoic acid, from an ethanolic plant extract, did not affect NO production in LPS-stimulated RAW 264.7 macrophages. ${ }^{33}$

According to the obtained results, none of the species showed anti-inflammatory activity. But, in previous studies presented by Moro et al. ${ }^{13}$ with methanolic extracts, A. bisporus showed an inhibition of NO production by $30 \%$ at $0.5 \mathrm{mg} \mathrm{mL} \mathrm{m}^{-1}$ and $P$. ostreatus $15 \%$ at $0.5 \mathrm{mg} \mathrm{mL} \mathrm{mL}^{-1}$. These authors explain that pyrogallol that is only present in extracts of A. bisporus, Cantharellus cibarius Fr., Craterellus cornucopioides (L.) Pers. and Lactarius deliciosus (L. ex Fr.) S.F. Gray cited by Palacios et al. ${ }^{13}$ may be partly responsible for the antiinflammatory activity. In the same way, pyrogallol from the medicinal plant Emblica officinalis has been identified as one of the active compounds responsible for the anti-inflammatory effect of E. officinalis extracts in bronchial epithelial cells. ${ }^{34}$ Synergisms between pyrogallol and other active compounds present in the extracts, such as flavonoids or cinnamic acid derivatives, could explain the differences in activities among the extracts studied by the mentioned authors. 
Table 3 Cytotoxic $\left(\mathrm{Gl}_{50}\right.$ values. $\left.\mu \mathrm{g} \mathrm{mL}^{-1}\right)$ and anti-inflammatory activity $\left(\mathrm{EC}_{50}\right.$ values. $\left.\mu \mathrm{g} \mathrm{mL}^{-1}\right)$ of the mycelia and culture media of $A$. bisporus and P. ostreatus

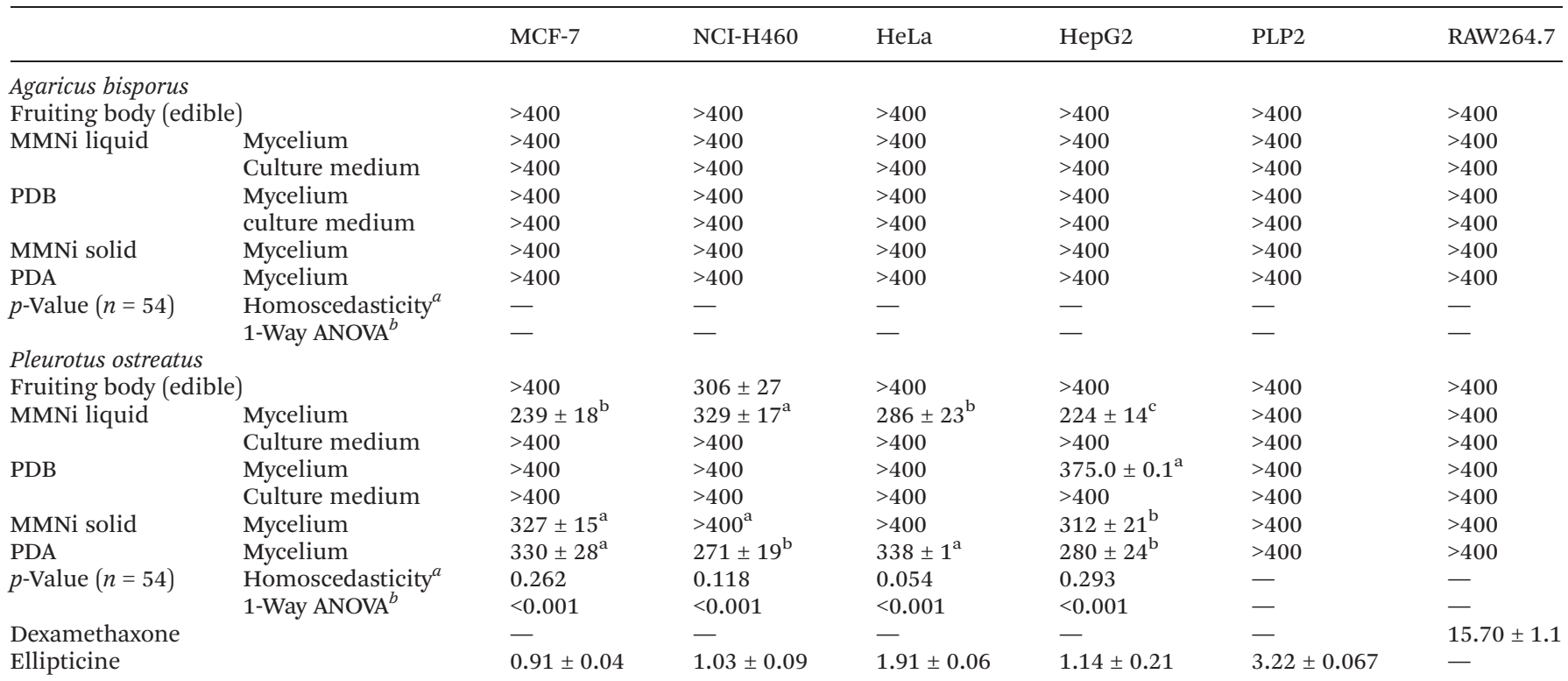

The values corresponding to the fruiting body of both mushrooms (edible samples) are also presented. Values are given as mean \pm standard deviation. ${ }^{a}$ Homoscedasticity among culture components was tested by the Levene test: homoscedasticity, $p>0.05$; heteroscedasticity, $p<0.05$. ${ }^{b} p<0.05$ indicates that the mean value of at least one component differs from the others (in this case, multiple comparison tests were performed). For each culture component, means within a column with different letters differ significantly $(p<0.05)$.

According to the results reported by Taofiq ${ }^{15}$ for ethanolic extracts ( $\mathrm{EC}_{50}$ values), P. ostreatus $\left(96 \pm 1 \mathrm{mg} \mathrm{mL}^{-1}\right)$ showed better results than A. bisporus $\left(190 \pm 6 \mathrm{mg} \mathrm{mL}^{-1}\right)$.

\subsection{Cytotoxic activity}

The cytotoxic activity of A. bisporus and P. ostreatus extracts against the four carcinoma cell lines (MCF-7, NCI-H460, HeLa and HepG2) and against a porcine liver primary cell culture (PLP2) is shown in Table 3.

As already stated, mushrooms are sources of several powerful new pharmaceutical products, in particular, and most importantly for modern medicine, representing an unlimited source of compounds with potential antitumor and immunostimulating properties. Many of these compounds have been used for the treatment of many health problems, including cancer. ${ }^{35}$

Only the extracts prepared from $P$. ostreatus samples showed some activity against the four tumor cell lines. A. bisporus extracts could not inhibit these cell lines (up to the maximum assayed concentration: $400 \mu \mathrm{g} \mathrm{mL}^{-1}$ extract), i.e., this species did not show any antitumor potential.

The extracts of $P$. ostreatus samples showed similar behavior in all cell lines $\left(\mathrm{GI}_{50}\right.$ values varying from $224 \pm 14$ to $375.0 \pm$ $0.1 \mu \mathrm{g} \mathrm{mL} \mathrm{m}^{-1}$ ). In this species, the fruiting body showed activity only for NCI-H460 $\left(\mathrm{GI}_{50}=306 \pm 27 \mathrm{mg} \mathrm{mL}^{-1}\right)$. The mycelium produced in MMNi liquid medium presented the highest cytotoxic effect against HepG2. All mycelia showed some activity in tumor cell lines, but none of the culture media showed activity.

A previous study reported the activities of A. bisporus and P. ostreatus mycelia (methanol extract) against HepG2 (54.9 \pm
2.4 and $56.3 \pm 2.5 \mathrm{mg} \mathrm{mL}{ }^{-1}$, respectively) and HeLa $(38.7 \pm 1.9$ and $52.2 \pm 2.5 \mathrm{mg} \mathrm{mL}^{-1}$, respectively). ${ }^{16}$

P. ostreatus samples that showed the highest cytotoxic activity also showed the highest contents of phenolic acids and ergosterol.

The extracts from A. bisporus and P. ostreatus did not exhibit a toxic effect on the non-tumor primary cell line. Interestingly, none of the assayed culture components inhibited the growth of the PLP2 cell line, which constitutes a good indicator for the lack of toxicity of the mycelia and culture media of both mushroom species in non-tumor cell lines.

Mushroom extracts can be used in combination with traditional chemotherapy or used as alternative sources for adjuvant cancer therapy, as some of these extracts have no adverse effects and activate the cells of the immune system. The bioactive compounds of mushrooms may complement classical cancer therapy and counter the side-effects of cancer, such as nausea, bone marrow suppression, anemia, and lowered resistance. ${ }^{36}$

\section{Conclusion}

This study was designed to develop nutraceutical formulations based on the mycelium of A. bisporus and P. ostreatus, as potential alternative sources of bioactive compounds or as ingredients to be used in applications with antioxidant, antiinflammatory or cytotoxic activities.

Overall, the growth rate of mycelia showed to be dependent on the culture media. Potato dextrose agar (PDA) medium was the best one for P. ostreatus, while solid modified Melin- 
Norkrans medium (MMNi) proved to be the best one for A. bisporus. The growth diameter rate was not correlated with the biomass of mycelium; for instance, radial growth was higher in solid than in liquid media for both species, but biomass production was higher in liquid than in solid media. We obtained better growth results in PDA and PDB media for $P$. ostreatus with similar biomass production, while $A$. bisporus exhibited similar biomass production in both media formulations but significantly higher under liquid conditions.

The methanolic extracts showed several phenolic acids ( $p$-hydroxybenzoic acid, $p$-coumaric acid and protocatechuic acid), a related compound (cinnamic acid) and ergosterol, which have been suggested to play an important role in antioxidant, anti-inflammatory and antitumor activities. In general, $P$. ostreatus mycelia showed higher contents of ergosterol. Cinnamic acid and $p$-hydroxybenzoic acid were also more abundant in the fruiting body of this species. A. bisporus mycelia produced in PDA showed higher amounts of phenolic acids and ergosterol, but $P$. ostreatus had higher contents of ergosterol, $p$-hydroxybenzoic acid and cinnamic acid in PDB and protocatechuic acid in PDA.

Likewise, the antioxidant activity was also higher among the $P$. ostreatus components. Extracts of A. bisporus did not show anti-inflammatory and cytotoxic activities up to the maximum assayed concentrations, in contrast to those observed for the mycelia of $P$. ostreatus, which showed some cytotoxicity, but also did not show anti-inflammatory activity. P. ostreatus mycelia showed cytotoxicity for tumor cell lines, which did not happen with the fruiting body. Furthermore, none of the species showed toxicity against non-tumor cells. The culture component showed differentiated activity, which should be considered together with the growth rate and biomass yielded for each mushroom.

\section{Conflict of interest}

There are no conflicts of interest to declare.

\section{Acknowledgements}

The authors are grateful to the Fundação para a Ciência e a Tecnologia (FCT, Portugal) for financial support to CIMO (strategic project PEst-OE/AGR/UI0690/2013) and for L. Barros and R. C. Calhelha contracts.

\section{References}

1 I. Siró, E. Kápolna, B. Kápolna and A. Lugasi, Appetite, 2008, 51, 456-467.

2 S. DeFelice, Trends Food Sci. Technol., 1995, 6, 59-61.

3 A. Cencic and W. Chingwaru, Nutrients, 2010, 2, 611-625.

4 L. Barros, M. Dueñas, I. C. F. R. Ferreira, P. Baptista and

C. Santos-Buelga, Food Chem. Toxicol., 2009, 47, 1076-1079.
5 I. C. F. R. Ferreira, L. Barros and R. M. V. Abreu, Curr. Med. Chem., 2009, 16, 1543-1560.

6 Z. Ren, Z. Guo, S. N. Meydani and D. Wu, J. Nutr., 2008, 138, 544-550.

7 C. Ramos, M. Sapata, A. Ferreira, L. Andrada and M. Candeias, Rev. Ciênc. Agrár., 2011, 34, 57-64.

8 A. Fernandes, L. Barros, A. Martins, P. Herbert and I. C. F. R. Ferreira, Food Chem., 2015, 169, 396-400.

9 D. Ren, N. Wang, J. Guo, L. Yuan and X. Yang, Carbohydr. Polym., 2016, 138, 123-133.

10 G. Ma, W. Yang, Y. Fang, N. Ma, F. Pei, L. Zhao and Q. Hu, LWT - Food Sci. Technol., 2016, 73, 108-116.

11 C. Zhang, S. Li, J. Zhang, C. Hu, G. Che, M. Zhou and L. Jia, Int. J. Biol. Macromol., 2016, 91, 568-577.

12 F. S. Reis, A. Martins, L. Barros and I. C. F. R. Ferreira, Food Chem. Toxicol., 2012, 50, 1201-1207.

13 C. Moro, I. Palacios, M. Lozano, M. D'Arrigo, E. Guillamón, A. Villares, J. A. Martínez and A. García-Lafuente, Food Chem., 2012, 130, 350-355.

14 A. Martins, E. Pereira, L. Barros and I. C. F. R. Ferreira, in 11 Encontro de Química dos Alimentos - Qualidade dos alimentos: novos desafios, Bragança, Portugal, 2012, pp. 1-5.

15 O. Taofiq, R. C. Calhelha, S. Heleno, L. Barros, A. Martins, C. Santos-Buelga, M. J. R. P. Queiroz and I. C. F. R. Ferreira, Food Res. Int., 2015, 76, 821-827.

16 A. Younis, J. Stewart, F.-S. Wu, H. E. Shikh, F. Hassan and M. Elaasser, Int. J. Sci. Technol., 2014, 3, 736-749.

17 N. G. Silva, Estudo da Capacidade Antioxidante de Cogumelos Comestíveis, Dissertação de Mestrado, Faculdade de Farmácia da Universidade de Coimbra, Coimbra, Portugal, 2015.

18 F. S. Reis, I. C. F. R. Ferreira, L. Barros and A. Martins, LWT - Food Sci. Technol., 2011, 44, 820-824.

19 M. Carocho, I. C. F. R. Ferreira, L. Barros, J. C. M. Barreira and A. Martins, Ind. Crops Prod., 2012, 38, 99-106.

20 S. A. Heleno, P. Diz, M. A. Prieto, L. Barros, A. Rodrigues, M. F. Barreiro and I. C. F. R. Ferreira, Food Chem., 2016, 197, 1054-1063.

21 S. A. Heleno, L. Barros, M. J. Sousa, A. Martins and I. C. F. R. Ferreira, Food Chem., 2010, 119, 1443-1450.

22 A. S. F. Fernandes, Escola Superior Agrária do Instituto Politécnico de Bragança, 2010.

23 L. Barros, C. Pereira and I. C. F. R. Ferreira, Food Anal. Methods, 2013, 6, 309-316.

24 R. M. V. Abreu, I. C. F. R. Ferreira, R. C. Calhelha, R. T. Lima, M. H. Vasconcelos, F. Adega, R. Chaves and M. J. R. P. Queiroz, Eur. J. Med. Chem., 2011, 46, 58005806.

25 R. Dal Toso and F. Melandri, Agro Food Ind. Hi-Tech, 2011, 22, 30-32.

26 A. Villares, L. Mateo-Vivaracho, A. García-Lafuente and E. Guillamón, Food Chem., 2014, 147, 252-256.

27 I. S. M. D. Falcão, Faculdade de Ciências da Universidade do Porto, 2008.

28 S. A. Heleno, L. Barros, A. Martins, M. J. R. P. Queiroz, C. Santos-Buelga and I. C. F. R. Ferreira, J. Agric. Food Chem., 2012, 60, 4634-4640. 
29 W.-J. Yoon, Y. M. Ham, S.-S. Kim, B.-S. Yoo, J.-Y. Moon, J. S. Baik, N. H. Lee and C.-G. Hyun, EurAsian J. Biosci., 2009, 143, 130-143.

30 N. H. Nam, Mini-Rev. Med. Chem., 2006, 6, 945-951.

31 G. Yuan, M. L. Wahlqvist, G. He, M. Yang and D. Li, Asia Pac. J. Clin. Nutr., 2006, 15, 143-152.

32 A. García-Lafuente, E. Guillamón, A. Villares, M. A. Rostagno and J. A. Martínez, Inflammation Res., 2009, 58, 537552.
33 M. Yu, X. Xu, Y. Qing, X. Luo, Z. Yang and L. Zheng, Eur. Food Res. Technol., 2009, 228, 477-485.

34 E. Nicolis, I. Lampronti, M. C. Dechecchi, M. Borgatti, A. Tamanini, N. Bianchi, V. Bezzerri, I. Mancini, M. Grazia Giri, P. Rizzotti, R. Gambari and G. Cabrini, Int. Immunopharmacol., 2008, 8, 1672-1680.

35 S. Wasser, Appl. Microbiol. Biotechnol., 2003, 60, 258-274.

36 S. Patel, D. Showers, P. Vedantam, T. R. Tzeng, S. Qian and X. Xuan, Biomicrofluidics, 2012, 6, 1-12. 